\title{
Etude hydrobiologique de la vallée d'Ossau (Pyrénées- Atlantiques) \\ II. Le milieu et la structure du peuplement benthique
}

\author{
G. Vinçon 1
}

Mots clés : Pyrénées, val lée d'Ossau, montagne, cours d'eau, faunistique, invertébrés benthiques, affinités cénotiques, zonation des eaux courantes.

Des récoltes d'invertébrés benthiques ont été effectuées dans le Gave d'Ossau, rivière des Pyrénées-Atlantiques, et dans ses principaux tributaires, entre 450 et $2150 \mathrm{~m}$ d'altitude. Après une présentation détaillée du milieu, un bilan des espè. ces les plus intéressantes est donne : 7 sont nouvelles pour la Science, 16 pour la France et 26 pour la zone 2 de la $\mathrm{Limno}$ fauna Europaea.

La succession longitudinale des biocénoses et leur déterminisme sont ensuite analysés. Sur le Gave d'Ossau, on observe un remplacement progressif des espèces d'amont par des espèces d'aval plutôt qu'une succession de communautés nettement individualisées ; le schéma de zonation des cours d'eau proposé par Illies et Botosaneanu (1963) apparaitt difficile à appliquer à ces rivières de montagne.

Hydrobiological study of the Ossau valley (Atlantic Pyrenees). II. The environment and the structure of the benthic population.

Keywords : Pyrenees, Ossau Valley, mountain, river, faunistic, benthic invertebrates, biocœnotic affinities, running water zonation.

Collections of benthic invertebrates have been taken in the Gave d'Ossau, a river in the Atlantic Pyrenees, and in its principal tributaries, between altitudes of $450 \mathrm{~m}$ and $2150 \mathrm{~m}$. After presenting a detailed description of the environment, a list of the most interesting species is presented : 7 species are new to science, 16 species are new records for France. and 26 species are new records for zone 2 in the Limnofauna Europaea.

The longitudinal succession of the biocœnoses and their composition have been analysed. In the Gave d'Ossau, there is a gradual replacement of upstream species by downstream species rather than a succession of strictly defined communities; the zonation scheme for running waters proposed by Illies \& Botosaneanu (1963) is apparently difficult to apply to mountain rivers.

\section{Introduction}

Dans les Pyrénées occidentales, l'étude du Gave d'Ossau et de ses principaux tributaires a été entreprise de septembre 1983 à novembre 1984 dans le but de tester une méthode biologique de surveillance des cours d'eau de montagne (Vinçon 1987 a et b).

Les 29 stations prospectées ont été choisies en vallée d'Ossau (27 stations échelonnées entre 450 et

1. Laboratoire d'Hydrobiologie, UA 695 C.N.R.S., Universite Paul Sabatier, 118, route de Narbonne, 31062 Toulouse Cedex, France.
$2150 \mathrm{~m}$ d'altitude) mais également dans la haute vallée du Rio Gallego, sur le versant espagnol (2 stations, à 1740 et $1750 \mathrm{~m}$ ), pour évaluer l'influence de l'exposition Sud sur les peuplements benthiques.

Auparavant, les cours d'eau des PyrénéesAtlantiques n'avaient jamais fait l'objet de recherches hydrobiologiques approfondies, à l'exception de l'étude d'une rivière côtière de basse altitude, la Nivelle, au-dessous de $50 \mathrm{~m}$ ( $\mathrm{fig}$. 1) et d'un de ses principaux affluents, le Lissuraga, entre 400 et $50 \mathrm{~m}$ (Thibault 1971 a, b et c, Neveu 1972, 1973 et 1980 , Tiberghien 1976, Lapchin \& Neveu 1979 a et b, Neveu, Lapchin \& Vignes 1979...). 


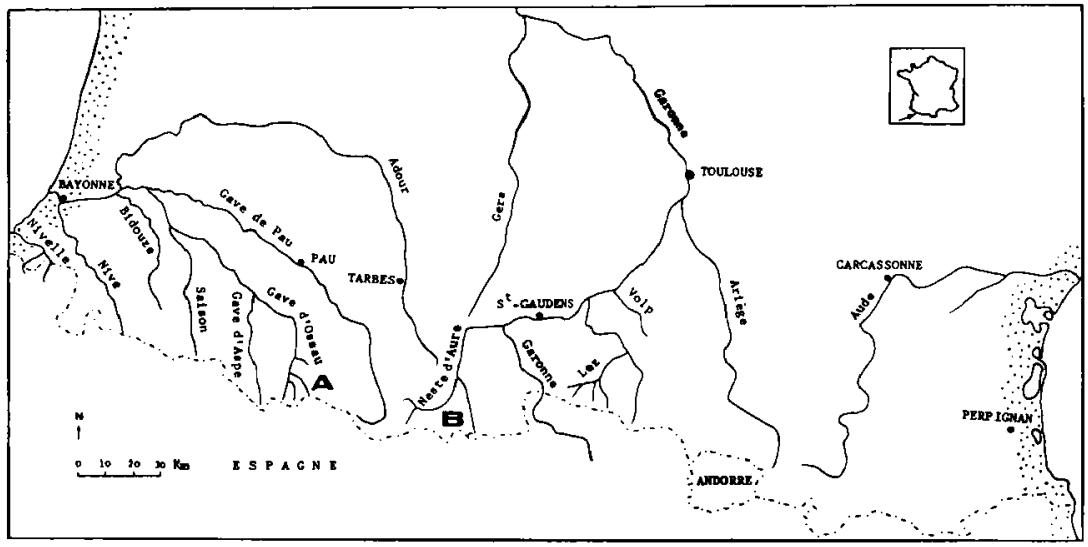

Fig. 1 : Localisation des deux vallées pyrénéennes: $\mathbf{A}=$ Gave d'Ossau et $\mathbf{B}=$ Neste d'Aure.

Le Gave d'Ossau ne sera pas comparé à ces deux cours d'eau de basse altitude mais à la Neste d'Aure (Pyrénées centrales) qui, de par sa situation en zone de haute montagne, lui ressemble davantage. C'est aussi la rivière la mieux connue des Pyrénées du point de vue hydrobiologique (inventaire bibliographique: Vinçon 1987a p. 30 et 1987b p. 12).

Dans ce travail, nous donnons une présentation détaillée de la vallée d'Ossau et des stations prospectées puis nous étudions la succession des biocénoses et leur déterminisme.

La distribution et l'écologie des principaux groupes systématiques feront l'objet d'études ultérieures. Certaines d'entre elles sont déjà réalisées : Vaillant \& Vinçon (1986 a et b) sur les Thaumaleidae et Empididae (Diptera) ; Vinçon \& Thomas (1987) sur les Ephéméroptères; Clergue-Gazeau \& Vinçon (en prép.) sur les Simuliidae (Diptera).

\section{Le milieu}

Les travaux de Poitrinal (1970). Lalanne (1976), Froelhy (1977) et Hourcade (1978) donnent une synthèse des caractéristiques écologiques de la vallée d'Ossau. Ici, nous présentons plus particulièrement le réseau hydrographique et les paramètres $d u$ milieu qui influent sur l'environnement du cours d'eau.

\subsection{Réseau hydrographique}

Le cours principal du Gave d'Ossau reçoit une douzaine d'affluents plus ou moins importants dont les principaux sont : les Gaves de Bious et de Brousset, le Soussouéou et le Valentin (fig. 2).

La surface de son bassin-versant est de $280 \mathrm{~km}^{2}$ au pont de Béost $(489 \mathrm{~m})$, ce qui est peu, comparé à celle de la Neste d'Aure $\left(660 \mathrm{~km}^{2}\right)$ au pont de Bizous ( $490 \mathrm{~m}$ ). En basse altitude, le débit du Gave d'Ossau sera donc moins important que celui de la Neste d'Aure (abstraction faite des eaux prélevées par le canal de la Neste).

Les cinq sommets culminants de la vallée d'Ossau ont une altitude moyenne de $2830 \mathrm{~m}$ et le réseau hydrographique est très peu étendu au-dessus de $2300 \mathrm{~m}$. En vallée d'Aure, par contre, l'altitude moyenne est nettement plus élevée $(3140 \mathrm{~m}$ ) et le réseau atteint des altitudes supérieures $(2750 \mathrm{~m}$ dans le torrent de Cap de Long).

Le profil en long de la vallée d'Ossau (fig. 3) met en évidence les particularités de certains affluents : 


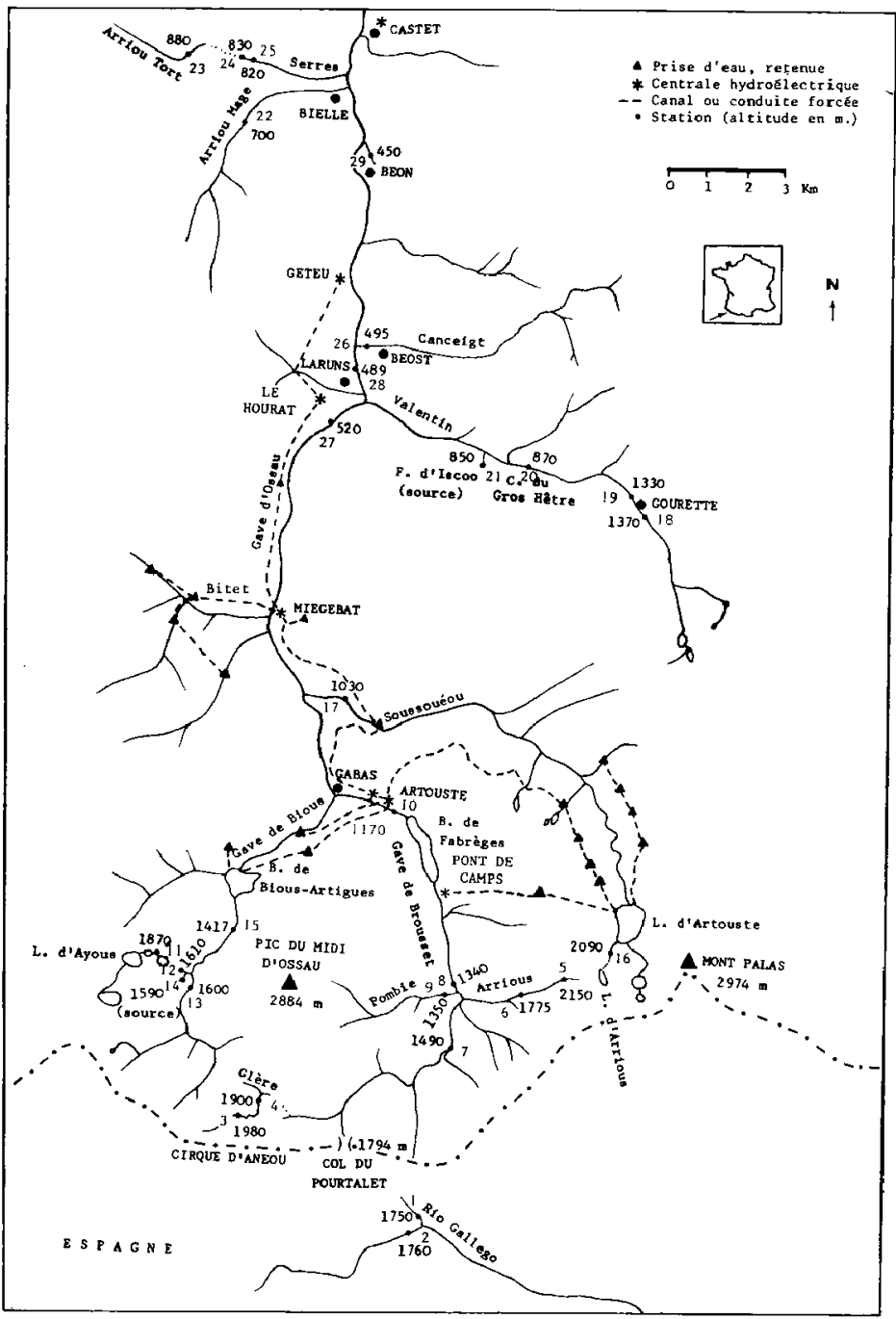

Fig. 2 : Réseau hydrographique du Gave d'Ossau. Emplacement des stations (avec leur altitude) et des principaux aménagements hydrauliques. 


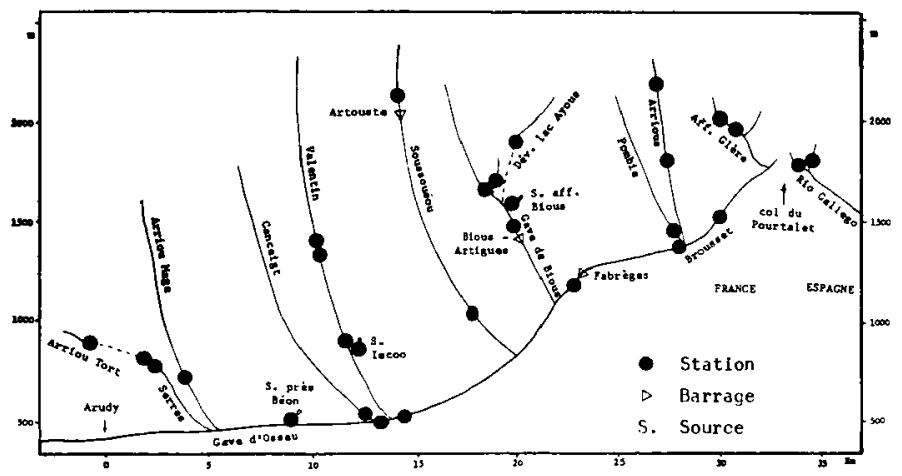

Fig. 3: Profil en long de la vallée d'Ossau.

1) le Soussouéou, le Valentin et le ruisseau d'Arrious sont issus de sources de très haute altitude $(>2200 \mathrm{~m}$ ) et ont une pente très raide dans leur secteur amont ; ce sont des cours d'eau relativement froids en été (\$ 3.2.) :

2) le ruisseau affluent de la Glère, le déversoir du lac d'Ayous et le Rio Gallego (Espagne) prennent leurs sources à des altitudes inférieures (1 800 $2000 \mathrm{~m}$ ), sont moins encaissés, avec des pentes plus modérées. De ce fait, ils sont caractérisés par des températures estivales beaucoup plus élevées (§3.2.) :

3) en zone de piémont ( $880 \mathrm{~m})$, l'Arriou Tort bénéficie aussi d'un régime thermique privilégié qui est la conséquence d'une pente quasi nulle et d'un courant très lent.

Les principaux aménagements (usines et barrages appartenant essentiellement à la S.N.C.F.) sont indiqués sur la figure 2.

\subsection{Influences climatiques}

Les précipitations augmentent avec l'altitude mais varient également avec l'orientation des bassinsversants. Ainsi, la moyenne vallée d'Ossau orientée au Nord reçoit 1200 à $1500 \mathrm{~mm}$ de précipitations par an alors que la moyenne vallée d'Aure orientée à l'Est et au Nord-Est en aval de Fabian n'en reçoit que 800 à $1000 \mathrm{~mm}$. Cette dernière est d'autre part protégée de l'influence des vents dominants humides du Nord et du Nord-Ouest par le massif montagneux qui s'étend du Pic du Midi de Bigorre (2 $872 \mathrm{~m}$ ) au Pic du Néouvielle (3 $091 \mathrm{~m}$ ) (effet orographique d'abri).

Le régime du Gave d'Ossau et de ses principaux affluents de haute altitude est de type nival de transition, caractérisé par des crues de fonte des neiges plus ou moins violentes, de la fin du printemps jusqu'au début de l'été.

\subsection{Végétation}

Malgré une influence océanique plus marquée en vallée d'Ossau qu'en vallée d'Aure, les répartitions altitudinales des cinq étages de végétation sont assez semblables (Vinçon 1987a, p. 36), avec successivement : l'étage atlantique $(<700 \mathrm{~m})$, collinéen $(700$ $1300 \mathrm{~m})$, montagnard (1 $300-1800 \mathrm{~m})$, subalpin $(1800-2300 \mathrm{~m}$ ) et alpin ( $>2300 \mathrm{~m})$. Cependant, en vallée d'Ossau, l'étage alpin couvre une surface net. tement plus réduite que dans les Pyrénées centrales, faute d'altitudes suffisamment élevées à l'ouest du col du Pourtalet.

\subsection{Géologie sommaire}

A l'exception du massif cristallin du Mont Palas drainé par le Soussouéou, la haute vallée d'Ossau (régions de Brousset et de Bious) est principalement constituée de terrains sédimentaires icalcaires, pélites et grès, ...) auxquels sont venues s'ajouter des 
intrusions d'origine volcanique, essentiellement des andésites basiques. Celles-ci proviennent de "l'épisode Ossau " caractérisé par un volcanisme calcoalcalin.

Ces terrains sont donc essentiellement de nature basique. Ils ont été remaniés ensuite par les glaciers de l'époque quaternaire (structure décrite par Henry 1968 , Bixel et al. $1983,1985, \ldots)$.

La moyenne vallée d'Ossau, quant à elle, est constituée de terrains sédimentaires (schistes, calcaires, gneiss, grès, ...) auxquels s'ajoutent des marnes en zone de piémont.

\subsection{Influences humaines}

La haute vallée d'Ossau (secteurs de Bious, Brousset et Artouste) est située à l'intérieur du Parc National des Pyrénées Occidentales, la basse et la moyenne vallée appartiennent à sa zone périphérique.

Les principales ressources économiques de la région sont l'agriculture, le tourisme et l'industrie hydro-électrique.

Agriculture : en montagne et piémont, les prairies sont surtout consacrées au pâturage extensif (moutons, vaches et chevaux) ; cette utilisation n'entraine apparemment aucune nuisance sur les cours d'eau.

Tourisme : plusieurs villages de vacances sont présents en vallée d'Ossau et notamment deux stations de sports d'hiver : Artouste et Gourette. Il est actuellement question de les relier en aménageant la moyenne vallée du Soussouéou (projet contesté : étude du C.T.G.R.E.F. 1977).

Le torrent du Valentin a été prospecté en amont et en aval de Gourette (st. 18 et 19) pour apprécier l'influence de la pollution ; celle-ci affecte prinçipalement les espèces fragiles (Ephémères, Plécoptères) (Vinçon 1987a). Par contre, en amont et en aval du village de Laruns (st. 27 et 28), le Gave d'Ossau présente un peuplement assez semblable.

Industrie hydro-électrique : les principaux aménagements (barrages d'Artouste, de Fabrèges et de Bious-Artigues) permettent de contrôler les débits du Soussouéou et des Gaves de Brousset et de Bious. Ces aménagements induisent des modifications importantes dans les peuplements benthiques, comme par exemple à l'aval du barrage de Fabrèges (Vinçon 1987a).

\section{Les stations}

La grande diversité des stations interdisait toute approche quantitative stricte. Toutefois, pour l'obtention de données faunistiques comparables, les prélèvements dans chaque station ont été effectués pendant des laps de temps identiques. D'après Macan (1957) et Hynes (1961), la récol te de matériel larvaire (Ephémères ou Plécoptères) pendant une période de temps déterminée, permet de bons résul. tats comparatifs.

Dans les stations de basse et moyenne altitude, les prélèvements ont été effectués à six périodes de l'année : mars, mai, juin, juillet, septembre et novembre. Dans les stations de haute altitude, les récoltes ont été entreprises en fonction du déneigement des ruisseaux : mai (st. 1, 2, 6 et 11), juin (st. 4) et juillet (st. 3,5 et 16).

Le matériel a été récolté par dérive (récoltes de $90 \mathrm{mn}$ ), par prélèvements benthiques ( $20 \mathrm{mn}$ ) et par chasses à vue et au filet entomologique $(60 \mathrm{mn})$.

\subsection{Paramètres}

Dans les figures 4 et 5 , les 29 stations sont désignées par un code indiquant les initiales du cours d'eau, l'altitude de la station et son numéro. Voici les abréviations utilisées pour la désignation des stations $(\mathrm{r} .=$ ruisseau, $\mathrm{t} .=$ torrent, riv. $=$ rivière $)$ :

Ar. $=r$. d'Arrious $;$ D.Ar. $=r$. déversoir du lac d'Arrious ; A.G. $=r$. affluent de la Glère $;$ D.Ay. $=t$. déversoir du lac d'Ayous en amont du lac Roumassot : A.R.G. $=r$. affluent du Rio Gallego: R.G. = Rio Gallego; A.Bi. = r. affluent de Bious $; B i .=t$. de Bious $;$ S.A.Bi. = source affluent de Bious; Br. = t. de Brousset; $\mathrm{V} .=t$, du Valentin; $P .=$ r. de Pormbie; $S .=$ t. du Soussouéou; A.T. $=$ r. d'Arriou Tort $;$ S.I. $=$ source d'Iscoo $;$ Se.$=r$. de Serres $;$ A.M. $=$ r. d'Arriou Mage ; $\mathrm{O} .=$ riv. d'Ossau ; $\mathrm{C} .=\mathrm{t}$. du Canceigt et R.N.B. = r. au nord de Béon.

Le tableau I récapitule Ies caractéristiques abiotiques principales des stations : altitude, altitude de la source la plus en amont sur le même cours d'eau, régime des eaux, surface du bassin-versant, numéro d'ordre du cours d'eau, pente, largeur moyenne du lit mineur, vitesse du courant, nature du substrat, température maximale, alcalinité totale, dureté calcique et conductivité.

Les mesures physico-chimiques sont réalisées en septembre (étiage) et se rapprochent des valeurs maximales; la température a été mesurée à différentes périodes de l'année (fig. 4). Les méthodes d'analyse utilisées sont exposées dans les 
Tableau I : Caractéristiques abiotiques des stations. $N=$ nival, $N T=$ nival de transition, $P N=$ pluvio-nival, $T R=$ très rapide, $R=$ rapide, $M=$ moyenne, $L=$ lente, $p=$ pierres, $s=$ sable, $l=$ limon $e t v=$ vase. Les initiales des stations sant expliquées au § 3.2.

\begin{tabular}{|c|c|c|c|c|c|c|c|c|c|c|c|c|c|c|}
\hline 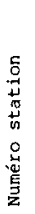 & 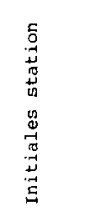 & 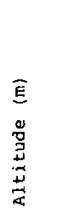 & 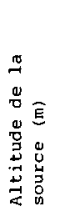 & 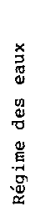 & 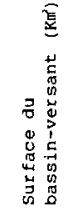 & $\begin{array}{l}0 \\
\mu \\
\stackrel{4}{0} \\
0 \\
0 \\
0 \\
0 \\
z\end{array}$ & $\begin{array}{l}\stackrel{\infty}{0} \\
\stackrel{0}{\stackrel{5}{0}} \\
\stackrel{0}{\omega}\end{array}$ & 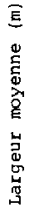 & 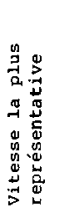 & 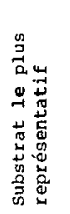 & $\begin{array}{l}\bar{u} \\
\dot{0} \\
\dot{x} \\
\dot{0} \\
\dot{0}\end{array}$ & 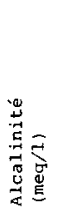 & 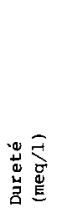 & 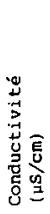 \\
\hline 1 & R.G. & 1750 & 1800 & $\mathrm{NT}$ & 0,3 & 9 & 5 & 2 & $M$ & $s+1$ & 15 & 1,19 & 1,56 & 172 \\
\hline 2 & $A \cdot R \cdot G=$ & 1760 & 1900 & $\mathrm{NT}$ & 0.8 & 1 & 10 & 3 & $\mathrm{R}$ & $\mathbf{p}$ & 15 & 0,53 & 0,68 & 75 \\
\hline 3 & A.G. & 1980 & 2080 & $\mathbf{N T}$ & 1,1 & 1 & 15 & 2 & $\mathrm{TR}$ & $\mathrm{p}$ & 15 & 0,26 & 0,44 & 52 \\
\hline 4 & $A . G$. & 1900 & 2080 & NT & 1,7 & 1 & 5 & 3 & $M$ & $p+s$ & 16 & 0.68 & 0,84 & 95 \\
\hline 5 & Af. & 2150 & 2230 & $\mathrm{~N}$ & 0,3 & 1 & 30 & 1 & M & $\mathrm{p}^{+\mathrm{s}}$ & 4,5 & 0,46 & 0.72 & 76 \\
\hline 6 & Ar. & 1775 & 2230 & $\mathrm{~N}$ & 2 & 2 & 10 & 3 & $\mathrm{R}$ & $\mathrm{p}$ & 9 & 0,61 & 0.76 & 84 \\
\hline 7 & $\mathrm{Br}$. & 1490 & 2080 & NT & 17 & 3 & 10 & 5 & $\mathrm{TR}$ & $\mathrm{p}$ & 14 & 1,21 & 1,48 & 163 \\
\hline 8 & $\mathrm{Br}$. & 1340 & 2230 & NT & 22 & 3 & 5 & 5 & $R$ & $\mathrm{p}$ & 13 & 1,00 & 1,20 & 139 \\
\hline 9 & P. & 1350 & 2000 & $\mathrm{NT}$ & 6 & 2 & 20 & 3 & TR & $\mathrm{p}$ & 12 & 0,50 & 0,64 & 73 \\
\hline 10 & $\mathrm{Br}$. & 1170 & 2230 & $\mathrm{NT}$ & 61 & 3 & 8 & 5 & $\mathrm{R}$ & $p+s$ & 13,5 & 1,20 & 1.30 & 156 \\
\hline 11 & D.AY = & 1870 & 2070 & NT & 1,8 & 2 & 25 & 3 & $\mathrm{TR}$ & $\mathrm{p}$ & 14 & 0,36 & 0,48 & 54 \\
\hline 12 & A.Bi . & 1610 & 1650 & NT & 2 & 1 & 30 & 3 & TR & $p$ & 16 & 0,38 & 0,44 & 55 \\
\hline 13 & $\mathrm{Bi}$. & 1600 & 2100 & NT & 12 & 3 & 20 & 3 & TR & $\mathrm{p}$ & 10 & 0,98 & 1,24 & 124 \\
\hline 14 & S.A.Bi & 1590 & 1590 & $\mathbf{s}$ & 0,2 & 0 & 20 & 2 & $M$ & $s+1$ & 6,5 & 1,40 & 1,60 & 184 \\
\hline 15 & $\mathrm{Bi}$. & 1417 & 2100 & NT & 18 & 3 & 15 & 5 & $\mathrm{TR}$ & $\mathrm{p}$ & 11 & 0,88 & 1.10 & 107 \\
\hline 16 & D.Ar. & 2090 & 2250 & $\mathrm{~N}$ & 0,2 & 1 & 3 & 2 & L & $p+s$ & 5 & 0,54 & 0.68 & 76 \\
\hline 17 & s. & 1030 & 2320 & $\mathbf{N T}$ & 52 & 3 & 15 & 6 & $\mathrm{TR}$ & $\mathrm{p}$ & 13 & 0,60 & 0.84 & 90 \\
\hline 18 & v. & 1370 & 2280 & NT & 7 & 3 & 10 & 4 & $\mathrm{TR}$ & $\mathrm{p}$ & 12 & $0,8 \mathrm{~B}$ & 1.08 & 82 \\
\hline 19 & v. & 1330 & 2280 & NT & 9 & 3 & 10 & 4 & $\mathrm{TR}$ & $\mathrm{p}$ & 12 & 1,30 & 1,60 & 168 \\
\hline 20 & v. & 870 & 2280 & NT & 16 & 3 & 5 & 4 & $\mathrm{R}$ & $\mathrm{p}$ & 13 & 1,47 & 1,70 & 195 \\
\hline 21 & S.I. & 850 & 850 & $\mathbf{S}$ & 0,3 & 0 & 30 & 2 & $\mathrm{R}$ & $\mathrm{p}$ & 8 & 1,86 & 2,12 & 218 \\
\hline 22 & A.M. & 700 & 1650 & $\mathrm{NT}$ & 15 & 2 & 15 & 2 & $\mathrm{TR}$ & $\mathrm{p}$ & 12 & 1,86 & 2,00 & 219 \\
\hline 23 & A.T. & 880 & 950 & PN & 9 & 1 & 1 & 2 & L & $v$ & 20 & $2,4 \mathrm{~B}$ & 2,48 & 283 \\
\hline 24 & Se. & 830 & 950 & $\mathbf{P N}$ & 9 & 1 & 2 & 2 & $L$ & $p+s+I$ & 16 & 3,68 & 3,88 & 402 \\
\hline 25 & Se. & 820 & 950 & PN & 9,5 & 1 & 10 & 2 & M & $p+s$ & 17,5 & 3,36 & 3,44 & 351 \\
\hline 26 & $c$. & 495 & 1650 & $\mathbf{N T}$ & 22 & 3 & 8 & 5 & $R$ & $\mathrm{p}$ & 15 & 1,94 & 2,64 & 287 \\
\hline 27 & 0 & 520 & 2320 & NT & 220 & 4 & 3 & 16 & $\mathrm{R}$ & $\mathrm{p}$ & 15 & 1,63 & 1,84 & 201 \\
\hline 28 & 0 & 489 & 2320 & NT & 280 & 4 & 1 & 18 & $\mathbf{R}$ & $\mathrm{P}$ & 15 & 1,63 & 1,80 & 190 \\
\hline 29 & R.N.B. & 450 & 500 & $\mathbf{s}$ & 0,2 & 0 & 2 & 2 & L & $p+s+v$ & 9 & 1,96 & 2,12 & 228 \\
\hline
\end{tabular}




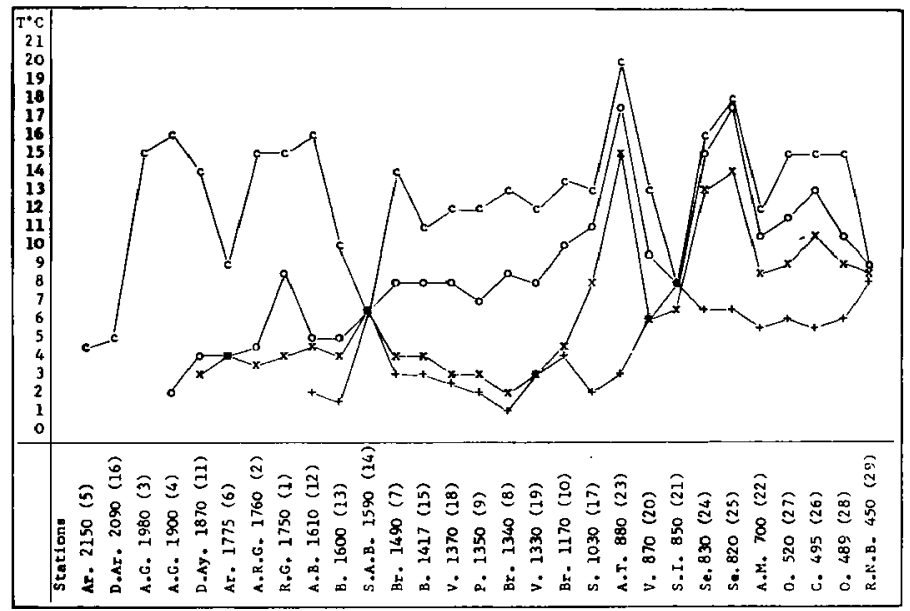

Fig. 4 : Températures mesurées dans les stations (classees par altitude décroissante), à 4 périodes de l'année : (+) du 16 au $22-03-$ 84, (x) du 30-04 au 3-05-84, (o) du 11 au 15-06-84, (c) du $27-07$ au 2-08-84. Les codes des stations sont expliqués au début du $\S 3.2$.

travaux de Lavandier et Mur (1974) et de Vinçon (1987a).

\subsection{Conclusion}

Si l'on exclut le massif du Néouvielle dont le réseau hydrographique atteint des altitudes très élevées, les vallées d'Aure et d'Ossau diffèrent assez peu sur l'ensemble des paramètres étudiés (climat, végétation, géologie, ...); elles se prêtent donc tout à fait à une comparaison de leurs peuplements respectifs.

Certains affluents du Gave d'Ossau possèdent cependant des caractéristiques particulières qui expliquent l'originalité de leur peuplement : le ruisseau presque stagnant d'Arriou Tort $(880 \mathrm{~m})$, le ruisseau calcaire de Serres ( 820 et $830 \mathrm{~m}$ ), le ruisseau au nord de Béon - résurgence karstique de pied de massif $(450 \mathrm{~m})$, assimilable à un crénal de basse altitude, ...

\section{Le peuplement : caractéristiques principales}

La liste faunistique globale comporte 516 taxons (447 identifiés à l'espèce) : elle est récapitulée sous forme de tableau à la fin de l'article, avec pour chaque espèce ses limites de répartition altitudinale et le nombre de stations où elle a été récoltée.

Grâce à la collaboration de nombreux spécialistes ( $\$ 6$ ), l'essentiel de la faune macroinvertébrée a pu être déterminé : Oligochètes (36 espèces), Hydracariens (48), Ephémères (30), Plécoptères (54), Coléoptères (35), Trichoptères (56), Diptères : Blephariceridae (5), Tipulidae (6), Limoniidae (12), Psychodidae (21), Dixidae (5), Simuliidae (27), Chironomidae (131), Thaumaleidae (4), Athericidae (2), Empididae (24), etc...

Les espèces les plus intéressantes d'un point de vue purement faunistique sont reportées dans le Tableau II ; 26 sont nouvelles pour la zone 2 de la Limnofauna Europaea (Pyrénées $>1000 \mathrm{~m}$ ), 25 pour les Pyrénées $(<1000 \mathrm{~m}), 16$ pour la France et 7 pour la Science.

Ces citations nouvelles traduisent, en fait, le degré plus ou moins poussé des investigations dans les différents groupes systématiques. Ainsi, les découvertes les plus originales concernent la famille des 
Tableau II : Vallée d'Ossau : inventaire des espèces nouvelles pour les Pyrénées ( $>$ ou $<1000 \mathrm{~m}$ ), la France ou la Science.

$+=$ espèce nouvelle pour la zone considérée; - = espèce déjà signalée dans l'une des deux zones altitudinales pyrénéennes.

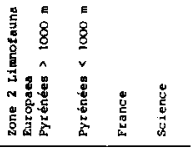

Ollgochetes

Stylodrilus? orachyetylus Hr.

stylodrilus parvus (Hr. S Cern.)

Haber pyrenaicus (Juget thiani)

Potamothr ix bevar feus (Osch.)

Spirosperma (E.) velutinus (Grube)

Ophidonais serpentina (Mäll.)

Cernosvitoviella n.sp.

Martonina argentea (Mich.,

Ephénér optères

caenis beskidensis sowa

Plécopticres

Leuctra n.sp.

Tr lchoptères

wormoldis txiangulifera mosely i ximins Annitella pyrenaea (Navas)

De ueus bolivari MoL.

Psychodidae

Panimerus gr. gressicus vaill.

Pericoma barbarica Vaill.

Pericoma calcilega feuerbotn

Pericoina fallax Eaton

Satchellielia pilularia (Tonn.)

vlomyia undulata (Tonn.)

Dixidae

Dixa nebuloga (keig.)

Simullidae

simulium (s.) gauaf Gren. \$ Faure

Ch1ronomidae

Diamese aberrata (Lundb.)

Odontonesa fulva ( $K$.)

Cerdiocledius capucinus lzett.

Chaetocladius melaleucus (Mg.)

Cricotopus lygropls Edw.

Euki efferiella fitkkaul tehm.

Heterotanytarsua aplcalis (R.)

Metriocnerus tristellus Edw.

orthocladius saxosus (Tok.)

Parametr iocnemus borecalpinus Grow.

Rheoemittia spinicornis Bx .

Thientemanniella acuticornis $\mathrm{K}$.

Thienemanniella vittata Edw.

Tvetenia veralls (5ow,)

Cryptochironorus aupplicana (wh.)

Stempellinella ninor (Btw.)

Thaum letdae

Thaumalea angelieri Vaill. - Vinç.

Empldidae

chelifera obscura vaill.

clinocera schremeri vaizl.

Clinocera stagnalis (Ha).)

clinocera subplectrum vaill. tvinc.

clinocera zwicki vaill. Einc.

phyllodromia melanocephala $F$.

wiedemannia ber thelemyi Vaili. sinc.

wiedemannis (Clinocerella) Sp.

wiedemannia (W.) digitata Vaill, Ging.

Totatr

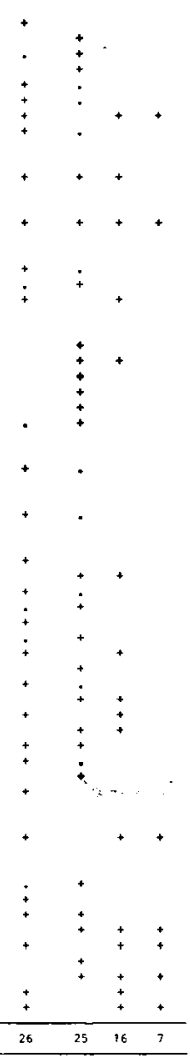

Diptères Empididae, encore insuffisamment connue dans les Pyrénées, avec un sous-genre (Clinocerella) nouveau pour la France et quatre espèces nouvelles pour la Science (Vaillant \& Vinçon 1986b).

Dans la famille des Chironomidae, un grand nom. bre de citations nouvelles correspond, le plus sou. vent, à des espèces rares ou sténotopes (Laville \& Vinçon 1986).

La plupart des espèces recensées ont une vaste répartition pyrénéenne (Vinçon 1987a, § 2.2.3.), ce qui confirme les observations de Berthélemy (1966) sur les Plécoptères et Coléoptères (Hydraena et Elmidae) : “ d'Est en Ouest, le peuplement des Pyrénées est assez homogene ".

\subsection{Richesse spécifique des stations}

Les peuplements les plus diversifiés se rencontrent dans les zones de moyenne montagne et de piémont. Ainsi, au-dessous de $1700 \mathrm{~m}$, une moyenne de 116 taxons a été récoltée dans chaque station (exclues les sources dont le peuplement est nettement moins diversifié) (fig. 5).

Dans les cours d'eau de haute altitude, la richesse spécifique décroit rapidement ; elle est en moyenne de 72 espèces dans les 8 stations situées au-dessus de $1700 \mathrm{~m}$. Cet appauvrissement peut être la conséquence de plusieurs facteurs : diminution des températures et des débits, enneigement prolongé, passage de la forêt montagnarde à la pelouse subalpine entrainant une diminution des ressources alimentaires exogènes...

\subsection{Les communautés et leur déterminisme}

\subsubsection{Méthode utilisée}

Pour mettre en évidence la succession des écosystèmes lotiques dans le réseau hydrographique du Gave d'Ossau, les peuplements des différentes stations ont été comparés deux à deux à l'aide de l'in. dice de similitude de $\mathrm{S}_{\boldsymbol{\phi}}$ rensen (1948) (Tableau III).

IS $=\frac{2 \mathrm{c}}{\mathrm{a}+\mathrm{b}}$

$\mathbf{a}$ et $\mathbf{b}$ : nombres d'espèces de chaque station. c : nombre d'espèces communes à deux stations.

Cet indice a été multiplié par 100 pour simplifier la présentation du tableau III.

Dans la préscnte étude, seuls sont pris en compte les Ephémères (30 espèces), les Plécoptères (54) et 
Tableau III : Affinités cénotiques (S $\phi$ rensen 1948) entre les stations (désignées par leur numéro et classées par altitude décroissante).

$A=$ ruisseaux froids à régime nival, au-dessus de $2000 \mathrm{~m} ; B=$ faciès torrenticoles de montagne et de piémont, à régime nival de transition ; $C=$ faciès lents, relativement chauds et à régime pluvio-nival.

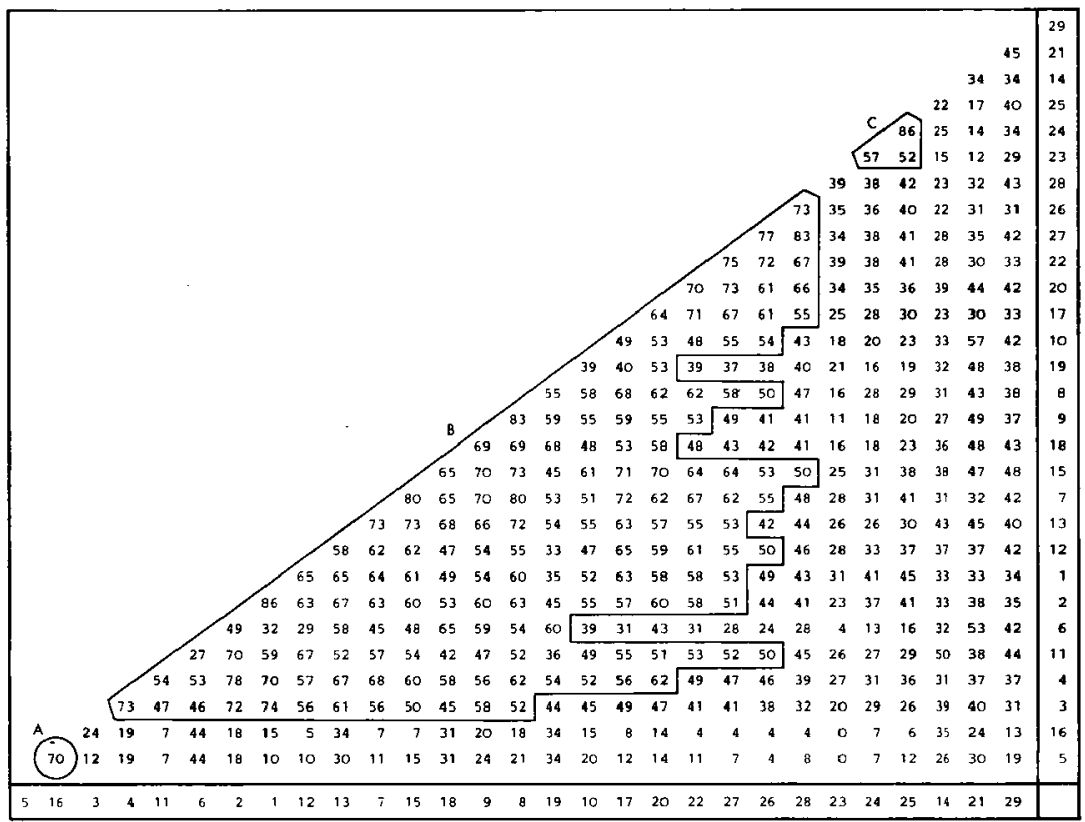

les Coléoptères Hydraena et Elmidae (21). Ces trois groupes d'insectes sont presque exclusivement diversifiés dans les biotopes de premier ordre situés dans l'axe du cours d'eau (pierres, strate muscinale, ...) et ont des préférendums écologiques différents, d'ou leur intérêt dans l'étude de la zonation des eaux courantes.

Pour faciliter la lecture du tableau III, nous avons isolé dans sa partie droite quelques stations particulières: le ruisseau lent d'Arriou Tort (st. 23), le ruisseau calcaire de Serres (st. 24 et 25) et les sources (st. 14, 21 et 29).

\subsubsection{Observations}

Le long du profil du Gave d'Ossau (Tableau IV), les affinités cénotiques (A.C.) entre 2 stations successives sont relativement fortes, ce qui traduit clairement la progressivité du remplacement des espèces d'amont par des espèces d'aval.

Les contrastes faunistiques les plus nets apparaissent non pas le long de ce profil mais entre certains affluents parfois peu éloignés.

D'après Angelier \& al. (1985), " parmi les facteurs écologiques qui sont à l'origine de la sélection de certains types biologiques, figure d'abord le régime des 
Tableau IV : Affinités cénotiques entre les 7 stations situées sur le profil en long du Gave d'Ossau.

Les valeurs A.C. les plus faibles s'expliquent par la présence d'un barrage $\left({ }^{\star}\right)$ et par une différence d'altitude importante $(* *)$.

\begin{tabular}{|c|c|c|c|c|c|c|c|}
\hline $\begin{array}{c}\text { Stations } \\
n^{\circ}\end{array}$ & 3 & 4 & 7 & 8 & 10 & 27 & 28 \\
\hline $\begin{array}{l}\text { Altitude } \\
\text { (m) }\end{array}$ & 1980 & 1900 & 1490 & 1340 & 1170 & 520 & 489 \\
\hline A.C. & & 73 & 68 & 80 & 58 & $\begin{array}{l}55 \\
\star \star *\end{array}$ & 83 \\
\hline
\end{tabular}

eaux ". Cette constation s'applique tout aussi bien aux Hydracariens étudiés par ces auteurs qu'au reste de la faune benthique; en effet, les stations les plus nettement individualisées sur le tableau III sont caractérisées avant tout par leur régime des eaux qui détermine principalement leur régime thermique.

Ainsi, en haute vallée d'Ossau, les ruisseaux froids à régime nival (st. 5 et 16) s'opposent à des ruisseaux à régime nival de transition, issus de sources de plus basse altitude et ayant des températures estivales nettement plus élevées (st. $3,4,11,2$ et 1); ces deux biotopes de haute montagne ont des peuplements très différents (A.C. <25). De tels contrastes faunistiques ont également été remarqués en haute vallée d'Aure, par exemple entre les torrents d'Estaragne et d'Estibère (Décamps 1967, p. 514).

De même, en basse et moyenne vallée d'Ossau, les torrents à régime nival de transition (st. 17 et 20) ont des peuplements assez différents de ceux des ruisseaux à régime pluvio-nival (st. 23,24 et 25) : A.C. $<36$ entre ces stations situées dans les mêmes tranches d'altitude.

Nous allons préciser maintenant le peuplement des principaux biotopes rencontrés en vallée d'Ossau. Certaines espèces sténotopes pourront aider à les caractériser.

a) Ruisseaux de haute montagne, froids, à régime nival

Dans une analyse factorielle des correspondances effectuée sur le groupe des Ephémères en vallée d'Ossau (Vinçon et Thomas 1987), ce biotope apparaît très nettement individualisé et est caractérisé par deux espèces sténothermes d'eau froide : $R$ hithrogena loyolaea et $R h$. strenua.

Ces ruisseaux pourraient correspondre aux " sommerkalten Bäche"des auteurs allemands (Brehm et Ruttner 1926). Leur peuplement s'appauvrit rapidement au fur et à mesure que les conditions de milieu deviennent plus sévères: 72 espèces à $1775 \mathrm{~m}$ (st. $6: \mathrm{T}^{\circ} \max .=9^{\circ} \mathrm{C}$ ); 55 à $2090 \mathrm{~m}$ (st. 16 : $\mathrm{T}^{\circ} \max .=5^{\circ} \mathrm{C}$ ) et 35 à $2150 \mathrm{~m}$ (st. $5: \mathrm{T}^{\circ} \max$. $=$ $4,5^{\circ} \mathrm{C}$ ).

Dans les biotopes aux conditions les plus extrêmes (st. 5 et 16), quelques groupes sont nettement dominants : les Oligochètes (12 espèces), les Plécop. tères (9), les Chironomidés (17) et les autres Diptères (19). Le groupe des Coléoptères, le moins diversifié, n'est représenté que par une espèce : Helophorus sp.

Le terme Kryon (Steffan 1971), utilisé pour décrire les biocénoses extrêmes, peut difficilement s'appliquer aux cours d'eau pyrénéens même les plus froids (Thomas 1981; p. 218); ils abritent en effet une majorité d'espèces du rhithron, à la limite de leurs possibilités d'adaptation et donc faiblement abondantes. Toutefois, en vallée d'Ossau, certaines espèces inféodées aux ruisseaux et sources situés au-dessus de $2000 \mathrm{~m}$ pourraient caractériser les biocénoses limites: Trichodrilus macroporophorus, Cer. nosvitoviella $n$. sp. (Oligochètes), Feltria minuta (Hydracarien), Leuctra alticola (Plécoptère), Thaumalea miki (Thaumaleidac), Bergenstammia pyrenaica et Clinocera zwicki (Empididae; Vaillant \& Vinçon 1986b).

b) Ruisseaux de haute montagne à températures estivales élevées et régime nival de transition.

Quelques ruisseaux de haute montagne à températures estivales élevées par rapport à leur altitude ont été étudiés dans les hautes vallées d'Ossau et du Rio Gallego (st. 3, 4, 11, 2 et 1; $\mathrm{T}^{\circ} \max .>15^{\circ} \mathrm{C}$ ). Selon la terminologie allemande, ils correspondent à des a sommerwarmen Bäche "(Brehm et Ruttner op. cit.).

Les paramètres qui favorisent le réchauffement de l'eau sont les suivants : débit réduit, pente faible et écoulement lent, situation abritée, exposition Sud (meilleure efficacite de l'ensoleillement et effet de Fö̈hn), absence de couvert végétal, présence d'un lac ou d'un marécage en amont, ... 


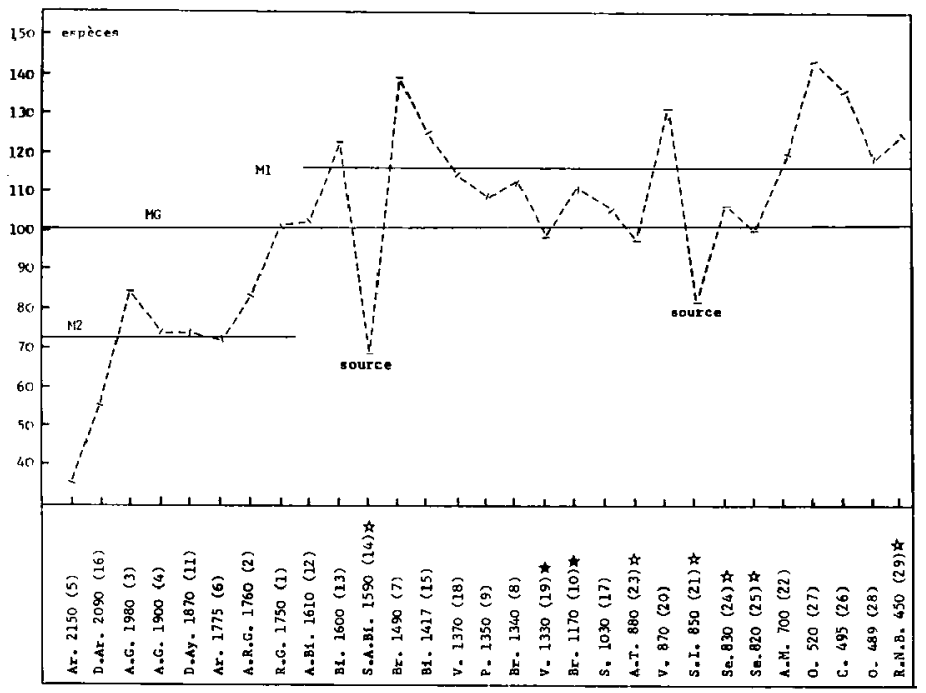

Fig. 5 : Richesse spécifique des stations (classées par altitude décroissante). $M G=$ moyenne générale, $M I=$ moyenne des stations situées au-dessous de $1700 \mathrm{~m}$ (sources exclues), $M 2=$ moyenne des stations situées au-dessus de $1700 \mathrm{~m}$.

Le peuplement de ces biotopes est très semblable à celui des cours d'eau de moyenne montagne comme on peut le voir sur le tableau III et comme nous l'avons remarqué aussi pour le groupe des Ephémères (Vinçon et Thomas 1987). On constate cependant une diminution progressive de la diversité spécifique allant de pair avec la réduction des dimensions du cours d'eau (fig. 5).

Grâce aux conditions thermiques favorables, cer. taines espèces d'aval atteignent dans ces biotopes leur limite supérieure de répartition altitudinale: Hygrobates calliger (Hydracarien), Simulium (S.) variegatum et $S$. (T.) bezzii (Simuliidae) ont été rencontrés à $1870 \mathrm{~m}$ dans le déversoir du lac d'Ayous (st. 11) : Baetis rhodani (Ephémère), Dinocras cephalotes et Perlodes microcephalus (Plécoptères) à $1900 \mathrm{~m}$ dans les méandres du ruisseau affluent de la Glère (st. 4) etc... c) Torrents de moyenne montagne $(800.1600 \mathrm{~m})$ d̀ régime nival de transition

Ces cours d'eau ont des caractéristiques écologiques intermédiaires entre celles des ruisseaux de haute altitude et celles des rivières de piémont. Leur peuplement peut donc être assimilé à celui d'un torrent de transition (Übergangsbach défini par Thienemann 1954). Ceci a aussi été constaté en moyenne vallée d'Aure (t 000 - $1500 \mathrm{~m}$ ) pour les Chironomidés (Gazagnes \& Laville 1985).

Ce peuplement de transition comprend: 1) des espèces eurythermes à large répartition al titudinale, souvent abondantes ; 2) quelques espèces davantage inféodées aux cours d'eau de moyenne montagne et susceptibles de caractériser ces biotopes : Rhithro. gena kimminsi (Ephémère), Brachyptera seticomis, Taenyopteryx hubaulti, Protonemura angelieri, Leuctra despaxi, Capnia nigra (Plécoptères); 3) des 
espèces sténothermes d'eau froide atteignant leur limite inférieure de répartition altitudinale (crénal excepté) : Rhithrogena loyolaea : $1330 \mathrm{~m}$, Rh. strenua : $1340 \mathrm{~m}$ (Ephémères), Protonemura tubercu. lata : $870 \mathrm{~m}$, Leuctra rauscheri : $1330 \mathrm{~m}$, Pachyle uctra benllochi: $1170 \mathrm{~m}$, Arcynopteryx compacta: $1030 \mathrm{~m}$, Isoperla viridinervis : $870 \mathrm{~m}$ (Plécoptères), Annitella pyrenaea : $870 \mathrm{~m}$ (Trichoptère), Diamesa hamaticornis : $870 \mathrm{~m}$, D. latitarsis : $1030 \mathrm{~m}$ (Chironomidae), etc.

Le développement plus ou moins important de cette communauté psychrosténotherme est étroitement lié aux conditions de milieu ; les facteurs qui peuvent induire un régime thermique froid sont les suivants : sources en très haute altitude, courant rapide, vallon encaissé et ombragé, sources rivulaires, glacier en amont,... (Lavandier 1974, Malicky $1978, \ldots)$.

d) Torrents et rivières de piémont à régime nival de transition

Leur peuplement est assez semblable à celui des torrents de moyenne montagne dont ils constituent le prolongement en basse altitude (Tableau III). Toutefois, quelques espèces peu alticoles et assez sténotopes peuvent les caractériser plus particulièrement : Sperchon clupeifer (Hydracarien), Ecdyonurus venosus (Ephémère), Isoperla grammatica sp. 2 (Plécoptère), Psychomyia pusilla (Trichoptère), Eukiefferiella ilkleyensis (Chironomidae)...

Dans ces biotopes, les Plécoptères sont en régres. sion et les Coléoptères en nette progression (Vinçon 1987 a, p. 230).

e) Ruisseaux de moyenne montagne $(800-900 \mathrm{~m}) \grave{a}$ régime pluvionival

Ils sont caractérisés par des sources situées audessous de $1000 \mathrm{~m}$ d'altitude, un courant lent ou modéré et des températures estivales élevées pour l'altitude ( $\mathrm{T}^{\circ} \max$. de 16 à $20^{\circ} \mathrm{C}$ ).

L'Arriou Tort à $880 \mathrm{~m}$ (st. 23), ruisseau lent à fond envasé et riche en macrophytes aquatiques, abrite un nombre élevé d'espèces thermophiles et léniticoles qui colonisent d'ordinaire les cours d'eau lents de plaine ou de piémont : Limnodrilus hoffmeisteri (Oligochète), Centroptilum luteolum, C. gr. pennulatum, Epheme rella major, Habrophlebia lauta, Paraleptophlebia submarginata (Ephémères), Hydraena pulchella, Oulimnius tuberculatus (Coléoptères), Anabolia nervosa, Goera pilosa, Mystacides nigra, Notidobia ciliaris, Beraeodes minutus (Trichoptères), Panimerus gr. gressicus (Psychodidae), Odontomesa fulva (Chironomidae), etc.

Le ruisseau calcaire de Serres à 820 et $830 \mathrm{~m}$ (st. 24 et 25 ) abrite un peuplement assez semblable (Tableau III) avec, en outre, plusieurs espèces réputées calcicoles: Stylodrilus lemani (Oligochète), Riolus subviolaceus (Coléoptère), Pericoma barbarica et $P$. calcilega (Psychodidae), Simulium (S.) gaudi (Simuliidae) et Lithotanytarsus emarginatus (Chironomidae).

Ces deux ruisseaux sont aussi caractérisés par un peuplement relativement riche en Ephémères et Diptères (Simuliidae, Chironomidae, ...) et, par contre, très pauvre en Plécoptères (Vinçon 1987 a, p. 230), groupe peu diversifié en faciès lent ou encroûtant.

\section{f) Sources}

L'eau froide des sources (résurgences karstiques) favorise le développement de certaines espèces alticoles en zone de piémont ou de moyenne montagne, ainsi Leuctra kempnyi, L. despaxi (Plécoptères), Diamesa latitarsis, Eukiefferiella fittkaui (Chironomidae) ont été rencontrées à $450 \mathrm{~m}$, dans un émissaire de sources froides (st. 29) et Pachyleucra benllochi, A rcynopteryx compacta et lsoperla viridinervis (Plécoptères) à $850 \mathrm{~m}$ dans la source d'Iscoo (st. 21).

De plus, la stabilité des conditions de milieu (faible amplitude thermique) influe sur les cycles de développement de certaines espèces qui ont tendance à se chevaucher au cours de l'année ; c'est le cas par exemple de Protonemura pyrenaica, Nernurella picteti et Nemoura erratica (Plécoptères) qui ont une période d'émergence particulièrement étalée dans le crénal (Vinçon 1987 a, p. 160).

En vallée d'Ossau, les espèces les plus crénophiles sont: Hydrovolzia placophora, Partnunia steinmanni (Hydracariens), Nemurella picteti (Plécoptère) et Ptychoptera albimana (Ptychopteridac).

\subsection{Correspondance de ces communautés avec les systèmes de zonation des eaux courantes}

Dans le réseau hydrographique du Doubs (Jura), Verneaux (1974) a différencié dix niveaux typologiques d'après les groupements d'espèces qu'tls abritent ; ils se remplacent progressivement de l'amont 
vers l'aval. D'après cet auteur, "l'absence de relations directes, simples et isolées entre espèces et composantes constitue un aspect fondamental de l'écologie ». De même, dans le réseau de l'Eau d'olle (Alpes du Dauphinè), Gay (1982) constate une évolu. tion continue du peuplement, de la source $(2000 \mathrm{~m})$ jusqu'à l'exutoire $(700 \mathrm{~m})$; le principal changement de faune n'étant pas dû à une brusque augmentation de débit mais à un ralentissement du courant dans la zone de piémont.

Certes, dans le Gave d'Ossau comme dans ces réseaux hydrographiques de haute montagne, des contrastes faunistiques assez nets peuvent apparaître en certains endroits particuliers d'un cours d'eau (confluents principaux, brusques variations de pente, ...) ; mais, d'un réseau à l'autre, ces disconti. nuités ne coïncident pratiquement jamais et il n'est donc pas possible de les choisir comme limites pour établir une zonation. Au contraire, on observe le plus souvent un remplacement progressif des espèces (§ 4.2.2.), si bien que le peuplement évolue de façon régulière et continue de l'amont vers l'aval.

En conséquence, le schéma proposé par Illies \& Botosaneanu (1963), faisant correspondre les changements de zones avec les confluents principaux, paraît difficile à appliquer aux torrents pyrénéens de haute montagne, comme l'a déjà fait remarquer Berthélemy (1966): " deux cours d'eau de même importance, mais situés à des altitudes différentes, peuvent présenter moins d'affinités faunistiques que deux portions du même cours d'eau, malgré une multiplication du débit par cinq ou six dans le second cas ». Cet auteur suggère d'accorder au débit et à l'altitude une impor tance égale pour caractériser les cours d'eau de haute montagne.

Ces observations montrent qu'il est difficile d'établir une zonation nette des cours d'eau pyrénéens. Ainsi, les ruisseaux de haute altitude, les torrents de transition et les rivières de piémont ne constituent pas trois zones écologiques nettement distinctes mais plutôt trois biocénoses se succédant progressivement.

Toutefois, des régimes hydrauliques et thermiques particuliers peuvent induire des contrastes faunistiques frappants même entre cours d'eau voisins et de dimensions semblables, comme nous avons $\mathrm{pu}$ le remarquer en haute vallée d'Ossau. Ceci justifie au moins la distinction de plusieurs types de cours d'eau suivant leur régime, nival, nival de transition, pluvio-nival, ... (\$ 4,2.2.).

En zone de montagne, le régime des eaux apparait donc utile pour différencier certains types biologiques (Angelier \& al. 1985, p. 62).

\section{Conclusion}

A la connaissance faunistique des écosystèmes lotiques des Pyrénées occidentales, ce travail apporte une contribution de 516 taxons récoltés dans 29 stations; c'est la première monographie d'un cours d'eau de montagne réalisée dans cette région.

A titre de comparaison, 594 espèces ont été recensées dans le réseau hydrographique de la Neste d'Aure (Pyrénées centrales), prospecté depuis plus de 25 ans et sans doute l'un des mieux connus d'Europe.

Les peuplements des vallées d'Aure et d'Ossau, comparés dans des études précédentes (Vinçon 1987 a et b), sont assez semblables; ce qui confirme l'homogénéité de la répartition des espèces aquatiques dans la partic centrale et occidentale des Pyrénées (Bertrand 1955, Berthélemy 1966).

Il serait intéressant d'étudier un réseau des Pyrénées-Orientales, cette région restant encore insuffisamment connue malgré quelques travaux récents : Décamps \& Pujol (1977), Moubayed (1986). Clergue-Gazeau (1987)... Ceci permettrait de préciser la répartition des espèces d'Est en Ouest de la chaîne pyrénéenne, d'évaluer l'influence des facteurs climatiques sur cette répartition et, peut-être, de mettre en évidence certaines barrières géographiques susceptibles d'empêcher la migration d'espèces (par exemple, les vallées de la Garonne, de l'Ariège, du Sègre, ...).

Les communautés d'espèces mises en évidence dans le réseau hydrographique du Gave d'Ossau à l'aide des affinités cénotiques entre stations ( $\$ 4.2$.) ont été confirmées, pour la plupart, dans l'analyse factorielle des correspondances effectuée sur les Ephémères (Vinçon \& Thomas 1987). Ces communautés ne permettent toutefois pas de proposer un schéma de zonation des cours d'eau de montagne. En effet, les ruptures de pente ou les confluents principaux peuvent constituer de bonnes limites entre les biocénoses d'un cours d'eau déterminé, mais la concordance entre les limites situées sur des cours 
d'eau différents n'a pas été établie (Berthélemy 1966). De l'avis de cet auteur, a l'étude de la sériation des Plécoptères dans les Pyrénées centrales et des Elminthidae en Europe n'apporte pas d'arguments en faveur de l'existence d'un nombre fixe de zones longitudinales, homologues d'un cours d'eau à l'autre".

\section{Remerciements}

La prospection de la vallée d'Ossau a été réalisée grâce à un financement de la Direction de la Protection de la Nature et grâce à l'aide technique du Parc National des Pyrénées Occidentales. Cette étude n'aurait pu être menée à bien sans l'aide des spécialistes qui ont identifié notre matériel : MM. N. Giani (Oligochètes), E. Angelier (Hydracariens), A. Thomas (Ephémères et certaines familles de Diptères), P. Zwick (Plécoptères), C. Berthélemy (Plécoptères et Coléoptères : Hydraena et Elmidae), G. Ferro (Coléoptères: Ochthebius), J.A. Régil-Cueto (Coléoptères : Hydrocanthares), H. Malicky (Trichoptères), J. Giudicelli (Diptères: Blephariceridae), Mme M. Clergue-Gazeau (Simuliidae), MM. B. Serra-Tosio (Chironomidae : Diame. sinae), H. Laville (autres Chironomidae) et F. Vaillant (Psychodidae, Thaumaleidae et Empididae). Nous les remercions tous vivement, ainsi que Mme $F$. Baurès qui a aidè à la frappe de cet article.

\section{Travaux cltes}

Angelier (E.), Angelier (M.L.) et Lauga (J.) 1985, - Recherches sur l'ecologie des Hydracariens (Hydrachnellae, Acari) dans les eaux courantes. Annls Limnol., 21 (1): 25-64.

Berthelemy (C.). 1966. - Recherches écologiques et biogeographiques sur les Plécoptères et Coléoptères d'eau courante (Hydraena et Elminthidae) des Pyrénées. Arnts Limmol, 2 (2) : 227.458 .

Bertrand (H.). 1955. - Sur la faune entomologique des Pyrénees. Verh. int. ver. theor. angew. Limnol, $12: 634.642$

Bixel (F.), Clin (M.). Lucas (C.), Majesté-Menjoulas (C.), Mirouse (R.) et Roger (P.). 1983 . - Pyrénées : 500 millions d'années. Itiné. raires géologiques dans le Parc National des Pyrénées. B.R.G.M. Orléans et P.N.P.O. Tarbes, 101 p. + i carte.

Bixel (F.), Muller (J.) et Roger (P.). 1985. - Carte géologique Pic du Midi d'Ossau et Haut Bassin du Rio Gallego. Inst. Géodyn. Univ. Bordeaux III. 54 p. +1 carte I.G.N.

Brehm (V.) et Ruttner (F.). 1926 - Die Biozönosen des Lunzer Gewässer. Internat. Rev. ges. Hydrobiol 16:330-392.

C.T.G.R.E.F. 1977 - La vallée du Soussouéou. Etudes préliminaires a la protection du site. Groupement de Grenoble. Division Protection de la Nature, étude $\mathrm{n}^{\circ}$ 115. $161 \mathrm{p}$.

Clergue-Gazeau (M.). 1987. - Influence du climat sur la faune simuliidienne (Diptera, Nematocera) d'une rivière pyrénéenne : la Massane. Ecologia Mediterranea, (sous presse).

Clergue-Gazeau (M.) et Vinçon (G.) 1988. - (à paraítre). Etude hydrobiologique de la Vallée d'Ossau (Pyrénées-Atlantiques) III. Les Simuliidae (Diptera, Nematocera) : leur originalité bio géographique et écologique. Annls Limnol.

Décamps (H.). 1967. - Ecologie des Trichoptères de la vallée d'Aure (Hautes-Pyrénées). Annis Limnol., 3 (3): 399.577

Décamps (H.) et Pujol (J.Y.). 1977. - Influences humaines sur le benthos d'un ruisseau de montagne dans les Pyrénées. Bull. Ecol., 8 (3): 349.358 .
Froelhy (F.). 1977. - Pyrénées des Gaves, eau et amenagement: Recherches bibliographiques 19291976. Thèse de 3ème cycle, Bordeaux, $304 \mathrm{p}$.

Gay (C.). 1982, - Les communautés benthiques d'un torrent des Alpes françaises : l'Eau d'Olle (1sère). Trav. Lab. Hydrobiol. Gre. noble: $7-31$.

Gazagnes (G.) et Laville (H.). 1985. - Etude faunistique des Chjronomidés (Diptera) de la Haute Neste d'Aure (Pyrénées Centrales) : impact des aménagements hydro-électriques. Annls Limnol., 21 (2): 149.159.

Henry (J.). 1968. - Itinéraires d'initiation à la géologie pyrénéerne. Vallée d'Ossau. Soc. Nat. des Pétroles d'Aquitaine. 16 p. +1 carte.

Hourcade (B.). 1978. - Ecologie de la vallée d'Ossau (Pyrénées occidentales). Recherches pour une synthèse (2 vol.). Edition du C.N.R.S. Bordeaux I.S.B.N. 222202322,240 p

Hynes (H.B.N.). 1961. - The invertebrate fauna of a welsh moun tain stream. Arch. Hydrobiol., $57: 344388$.

Illies (J.) et Botosaneanu (L.). 1963. - Problèmes et méthodes de la classification et de la zonation écologique des eaux courantes considérées surtout du point de vue faunistique. Mitt. int. Vereir. theor. angew, Limnol., 12 : 1.57

Lalanne (A.). 1976. - Ecologie et production forestiè res en haute vallée d'Ossau (Pyrénées-Atlantiques). D.E.A., Bordeaux, 35 p.

Lapchin (L.) et Neveu (A.). 1979a. - Hydrographie, climatologie et hydrologie de la Basse Nivelle, petit fleuve côtier des Pyrénées. Btill. Cent. Etud. Rech. Sci., Biarritz, 12 (4): $711-744$.

Lapchin (L.) et Neveu (A.). 1979b. - Ecologie des principaux invertébrés filtreurs de la Basse Nivelle (Pyrénées-Atlantiques). II : Hydropsychidae (Trichoptera). Annls Limnol., 15 (2) : 139-153.

Lavandier (P.). 1974. - Ecologie d'un torrent pyreneen de haute montagne I. Caractéristiques physiques. Annls Limnol., 10 (3) 173-219.

Lavandier (P.) et Mur (C.). 1974. - Ecologie d'un torrent pyrénéen de haute montagne. II - Caractéristiques chimiques. Annis Lim nol., 10 (3) : 275-309

Laville (H.) et Vinçon (G.). 1986. - Inventaire 1986 des Chironomidés (Diptera) connus des Pyrénées. Annls. Limnol, 22 (3) 239.251.

Macan (T.T.). 1957, - The Ephemeroptera of a stony stream. $J$. Anim. Ecol., 26 : 317-342.

Malicky (H.). 1978. - Der Temperaturgang einiger niederösterreichisher Gebirgsbäche (Ein Beitrag zur Typologie der Fliessgewässer). Weti. Leben (suppléments), 30:170-183.

Moubayed (Z.). 1986. - La rivière Massane (Pyr. Or.). Inventaire faunistique et recherches écologiques. Laboratoire Arago. Bagnuls-sur-Mer, $61 \mathrm{p}$.

Neveu (A.). 1972. - Introduction à l'étude de la faune des Diptères à larves aquatiques d'un ruisseau des Pyrénées-Atlantiques, le Lissuraga. Ann. Hydrobiol., 3 : 173-196.

Neveu (A.). 1973, - Le cycle de developpement des Simuliidae (Diptera. Nematocera) d'un ruisseau des Pyrénées-Atlartiques, le Lissuraga. Ann. Hydrobiol., 4 (1) : 51-75.

Neveu (A.), 1980. - La dérive des invertébrés aquatiques et terrestres dans un petit fleuve cótier de l'ouest des Pyrénées, la Nivelle. Acta Oecologica Oecol. Applic., 1 (4) : $317-339$

Neveu (A.), Lapchin (L.) et Vignes (J.C.). 1979. - Le macrobenthos de la Basse Nivelle, petit fleuve cótier des Pyrénées-Atlantiques. Ann Zool. Ecol anim. I1 (1): 85-111.

Poitrinal (D.). 1970. - Contribution à l'étude hydrologique et géo chimique de quelques bassins-versants de la chaine primaire axiale pyrénéenne. Thèse de 3ème cycle. Bordeaux, $n^{\circ} 816,111$ $p+$ figures et planches.

S $\phi$ rensen (T.). 1948. - A method of establishing groups of equal amplitude in plant sociology based on similarity of species content and its application to analyses of the vegetation on Danish commons. Biol. Skr., $5: 1-34$

Steffan (A.W.). 1971. - Chironomid (Diptera) bioccenoses in Scandinavian glacier brooks. Cam. ent., 103: 477-386 
Thibault (M.). 1971a. - Ecologie d'un ruisseau a truites des Pyrénées-Attantiques, le Lissuraga. I. Etude critique du milieu. Ann Hydrobiol, 2 (2) : 209.239.

Thibault (M.). 1971b. - Ecologie d'un ruisseau a truites des Pyrénées-Atlantiques, le Lissuraga. II. Les fluctuations thermiques de l'eau. Répercussion sur les perriodes de sortie et la taille de quelques Ephémeroptères, Plécoptè res et Trichoptères. Ann. Hydrobiol., 2 (2): $241-274$.

Thibault (M.). 1971c. - Le développement des Ephéméroptères d'un ruisseau à truites des Pyrénées-Atlantiques, le Lissuraga. Annls Limnol., 7 (1): 53-120.

Thienemann (A.). 1954. - Chironomus, Leben, Verbreitung und wirtschafliche bedeutung der Chironomiden. Binnengewässer. $20: 834 p$.

Thomas (A.G.B.). 1981. - Travaux sur la taxonomie, la biologie et l'ecologie d'insectes torrenticoles du Sud-Oue st de la France (Ephéméroptères et Diptères : Dixidae, Cecidomyiidae, Rhagionidae et Athericidae), avec quelques exemples de perturbations par l'homme. Thèse de Doctorat d'Etat, Toulouse, n ${ }^{\circ} 988.330 \mathrm{p}$. Tiberghien (G.), 1976. - Ecologie des Elodidae, Elminihidae, Hydraenidae d'un cours d'eau des Pyrénées-Arianiques: le Lissuraga. Thèse Doct. Univ. Toulouse, $440 \mathrm{p}$.
Vaillant (F.) et Vinçon (G.). 1986a. - Une espece nouvelle de Dip tères Thaumaleidae des Pyrénées. Annls Limnol., 22 (1): 65-68.

Vaillant (F.) et Vinçon (G.). 1986b. - Quelques Clinocerini (Diptera Empididae Hemenodromiinae) nouveaux ou mal connus des Pyrénées. Annls Limnol., 22 (3) : 261.275.

Verneaux (J.). 1974. - Recherches écologiques sur le réseau hydrographique du Doubs. Essai de Biotypologie. Thèse Besançon, $300 \mathrm{p}$.

Vinçon (G.) 1987a - Comparaison de la faune benthique des vallees d'Aure et d'Ossau, en vue de l'élaboration d'une méthodologie de surveillance des cours diean de montagne. Thèse Docteur-Ingénieur, Toulouse, $n^{\circ} 960.381 \mathrm{p}$.

Vinçon (G.) 1987b. - Cours d'eau de montagne. Deux méthodes de surveillance de leur qualité. Documents scientifiques du Parc National des Pyrénées, $n^{\circ} 23,61 \mathrm{p}$.

Vinçon (G.) et Thomas (A.G.B.). 1987. - Etude hydrobiologique de la Vallée d'Ossau (Pyrénees-Atlantiques). I. Répartition et écologie des Ephéméroptères. Annls Limnol., 23 (2) : 95-113. 


\begin{tabular}{|c|c|c|c|c|c|c|c|c|}
\hline & alt I tude & $N$ & & altitude & $N$ & & altitude & $N$ \\
\hline 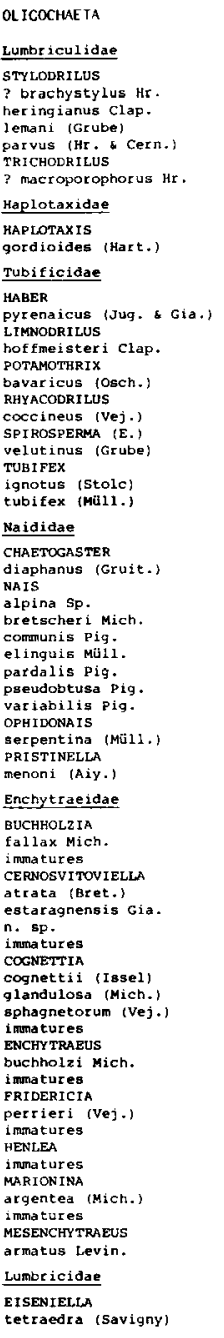 & $\begin{array}{l}490 \\
700-1600 \\
1750-2090 \\
1350-1760 \\
2090 \\
450-2150 \\
1330-1900 \\
1370-2090 \\
1420-2090 \\
870-2150 \\
500 \\
450-1775 \\
1610 \\
450-2090 \\
500-1980 \\
1490-1600 \\
880-1870 \\
450-2150\end{array}$ & $\begin{array}{r}1 \\
4 \\
3 \\
3 \\
1 \\
14 \\
2 \\
5 \\
2 \\
7 \\
1 \\
9 \\
1 \\
17\end{array}$ & 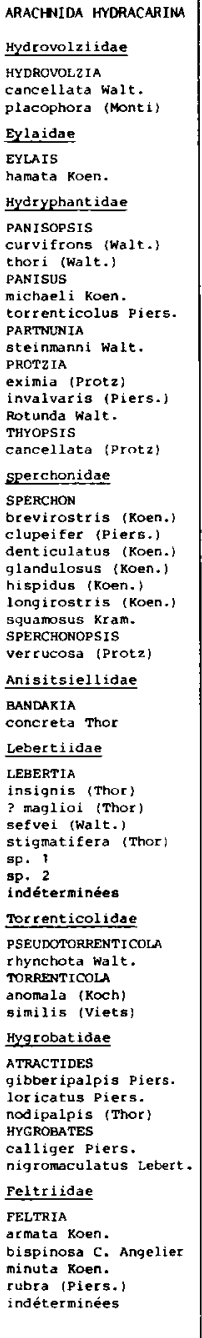 & $\begin{array}{l}500-1600 \\
500-1870 \\
450-1900 \\
490 \\
1590 \\
1420-1760 \\
450-1775 \\
700 \\
830\end{array}$ & $\begin{array}{r}8 \\
13 \\
1 \\
1 \\
2 \\
2 \\
10\end{array}$ & 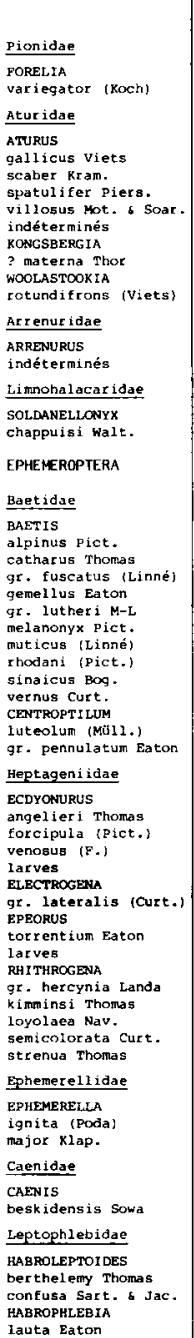 & $\begin{array}{l}700-1760 \\
1330-2090 \\
490-870 \\
450-2090 \\
1170-1980 \\
520-1870 \\
490-1870 \\
490-1980 \\
1340 \\
1330-2150 \\
450-1980 \\
1340-2090\end{array}$ & $\begin{array}{r}28 \\
13 \\
13 \\
21 \\
2 \\
13 \\
22 \\
21 \\
2 \\
1\end{array}$ \\
\hline
\end{tabular}




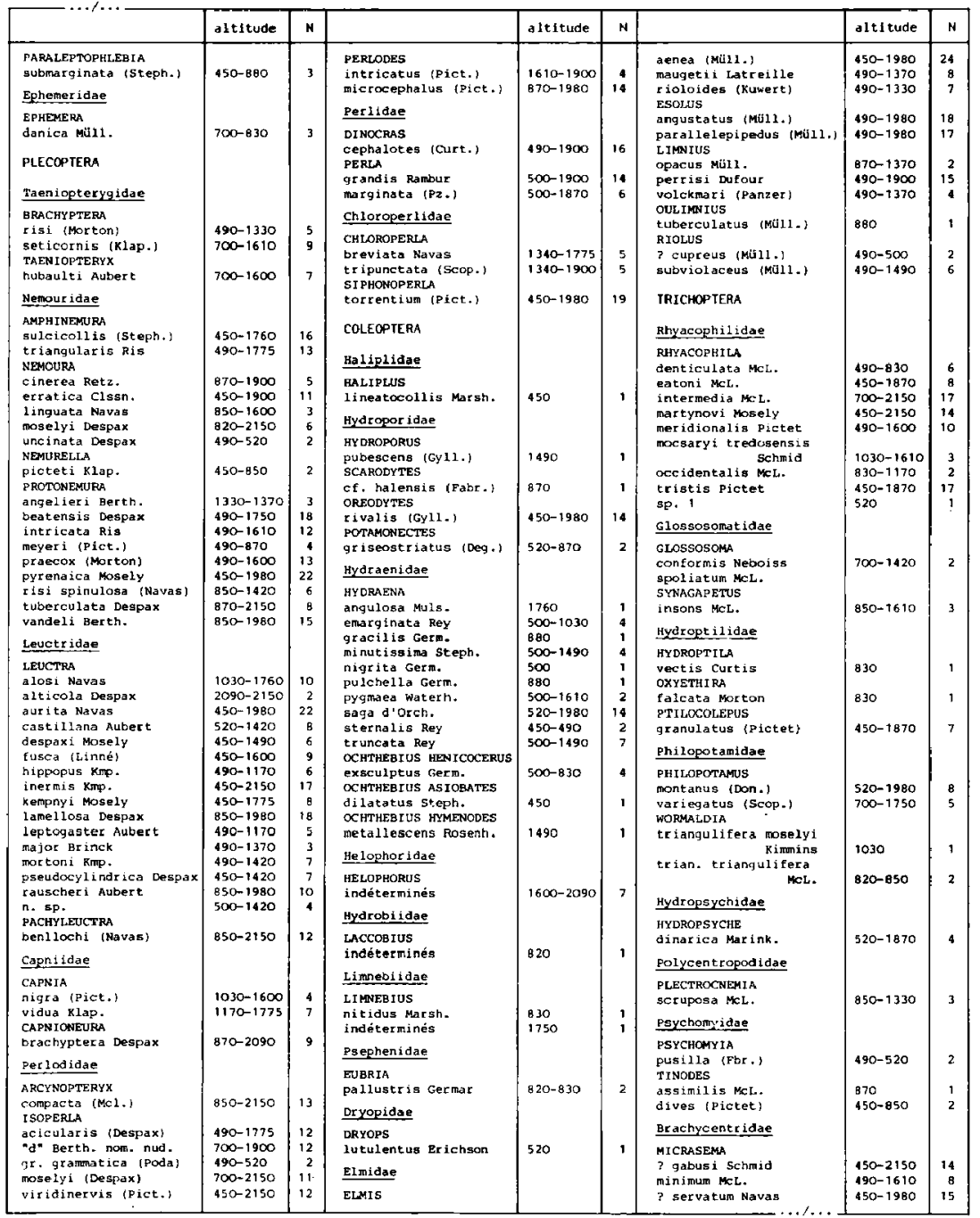




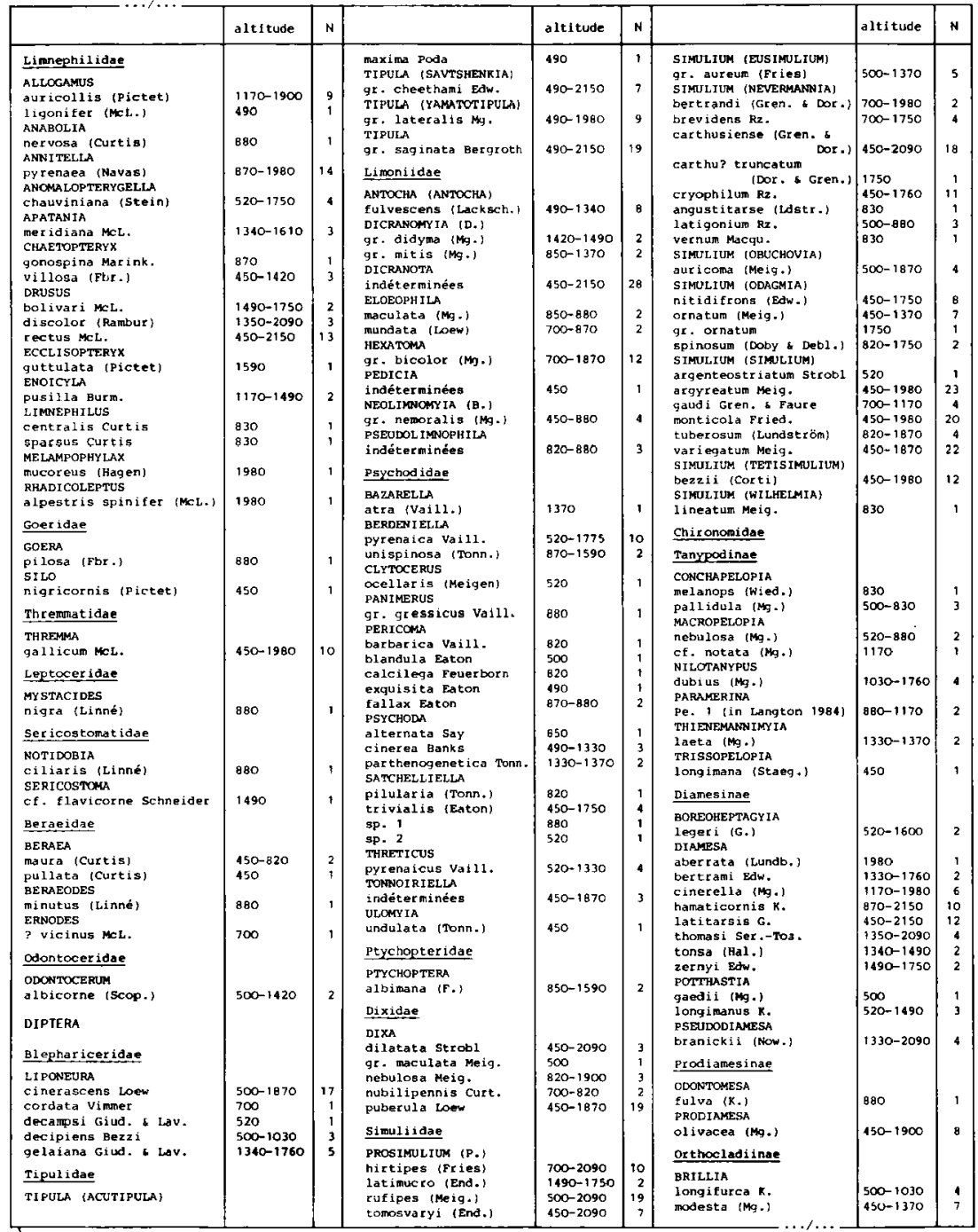




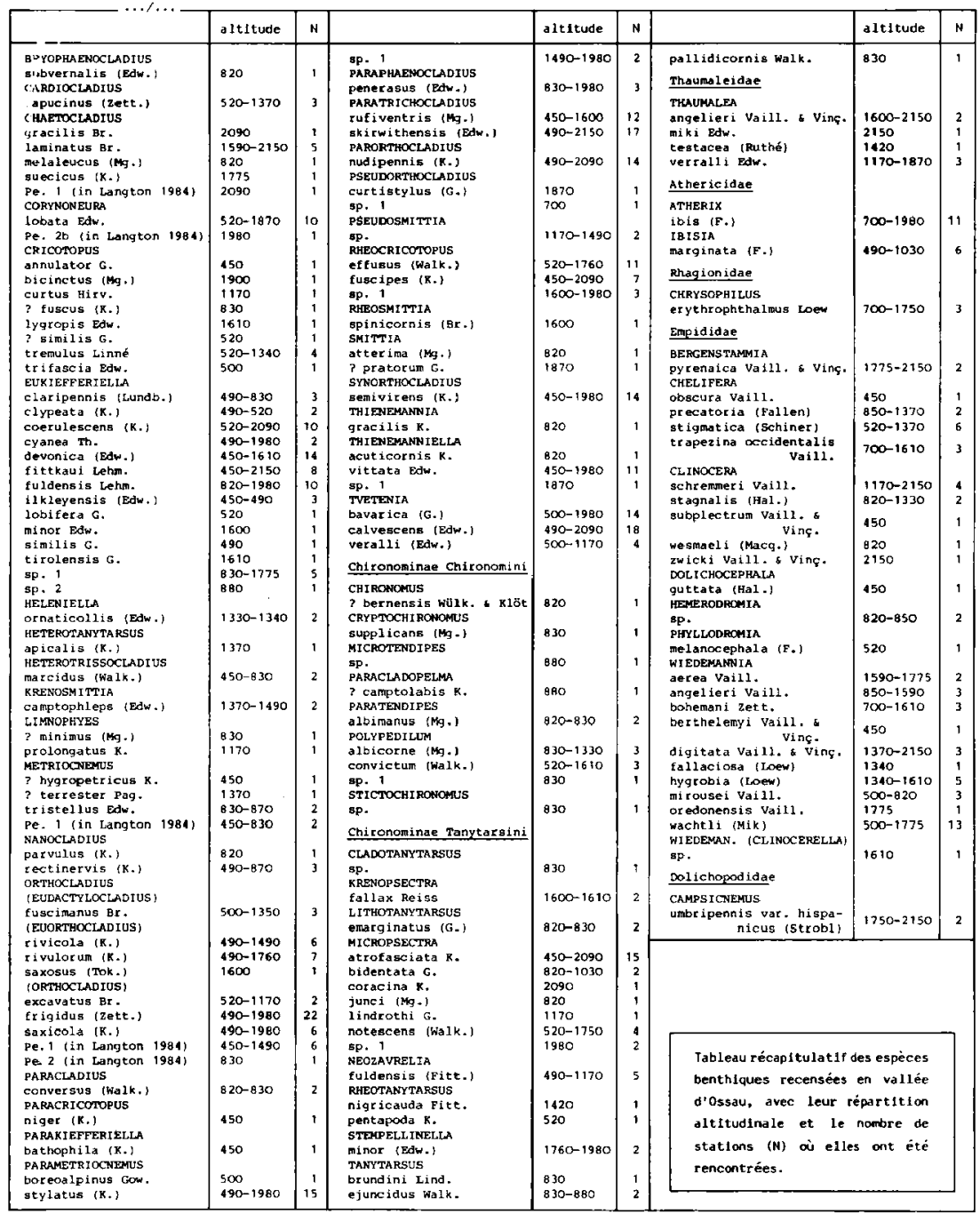

